\title{
Thermoplasmonic-Assisted Cyclic Cleavage Amplification for Self-Validating Plasmonic Detection of SARS-CoV-2
}

Guangyu Qiu, Zhibo Gai, Lanja Saleh, Jiukai Tang, Ting Gui, Gerd A. Kullak-Ublick,* and Jing Wang*

Cite This: ACS Nano 2021, 15, 7536-7546

Read Online

ACCESS | Lلll Metrics \& More | 回 Article Recommendations | st Supporting Information

ABSTRACT: The coronavirus disease 2019 (COVID-19) has penetrated every populated patch of the globe and sows destruction in our daily life. Reliable and sensitive virus sensing systems are therefore of vital importance for timely infection detection and transmission prevention. Here we present a thermoplasmonic-assisted dual-mode transducing (TP-DMT) concept, where an amplification-free-based direct viral RNA detection and an amplification-based cyclic fluorescence probe cleavage (CFPC) detection collaborated to provide a sensitive and self-validating plasmonic nanoplatform for quantifying trace amounts of SARS-CoV-2 within $30 \mathrm{~min}$. In the CFPC detection, endonuclease IV recognized the synthetic abasic site and cleaved the fluorescent probes in the hybridized duplex.

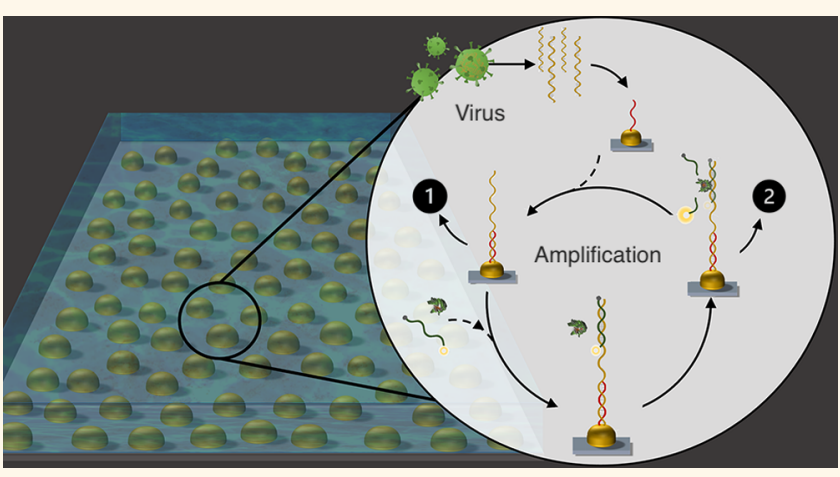
The nanoscale thermoplasmonic heating dehybridized the shortened fluorescent probes and facilitated the cyclical bindingcleavage-dissociation (BCD) process, which could deliver a highly sensitive amplification-based response. This TP-DMT approach was successfully validated by testing clinical COVID-19 patient samples, which indicated its potential applications in fast clinical infection screening and real-time environmental monitoring.

KEYWORDS: thermoplasmonics, SARS-CoV-2, COVID-19, plasmonics, biosensor, cyclic cleavage amplification

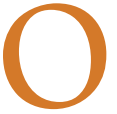

ver the past year, the coronavirus disease 2019 (COVID-19) pandemic caused by the severe acute respiratory syndrome coronavirus 2 (SARS-CoV-2) has penetrated every populated patch of the globe and sows destruction in daily life all around the world. ${ }^{1}$ This ongoing pandemic has engendered more than 113 million cases of infection and 2.5 million fatalities globally (as of February 27, 2021). ${ }^{2}$ Before effective vaccines are released to the general public, wide-scale testing and social distancing are still the most effective way to mitigate the spreading of SARS-CoV-2.,4 The wide-scale viral testing should not only encompass the surveillance test of suspected patients and the general public but also involve monitoring of the daily environment. ${ }^{5}$ In the infection screening and surveillance test, a highly sensitive diagnostic system could provide reliable results and triage people suspected of SARS-CoV-2. ${ }^{6}$ Meanwhile, a sensitive biosensing system can also be used for environmental monitoring with auxiliary modules for on-site sample collection, enrichment, and detection, so as to provide timely alerting as to the presence of the virus. ${ }^{7-9}$ Currently, polymerase chain reaction (PCR) is able to detect extremely low amount of viruses by means of nucleic acid (NA) amplification and has been utilized in clinical COVID-19 diagnosis as a "gold standard" ${ }^{10,11}$ Generally, the PCR technology requires sample pretreatments and usually inflexible desktop detection facilities; thus it has a limited role in real-time and point-of-care (POC) detection. ${ }^{11,12}$

Several biosensing systems for SARS-CoV-2 POC detection have been developed. According to the sensing targets, these biosensing approaches can be divided into two categories: viral NA-based detection and the viral protein-based detection (e.g., the spike protein). ${ }^{13-16}$ Specific protein-based immunodetection is the most straightforward way to identify the presence of pathogens, and this concept can be combined with different biosensing platforms, including the field-effect transistor $(\mathrm{FET})$ biosensor, ${ }^{17}$ plasmonic biosensors, ${ }^{18,19}$ and lateral

Received: February 1, 2021

Accepted: March 10, 2021

Published: March 16, 2021 
flow assay (LFA). ${ }^{20,21}$ By contrast, viral NA-based biosensors detect a particular viral NA sequence, which in principle have better specificity. ${ }^{22}$ For instance, the SHERLOCK (specific high-sensitivity enzymatic reporter unlocking) method and the DETECTR (DNA endonuclease-targeted CRISPR trans reporter) method based on CRISPR-Cas12 or CRISPRCas 13 have been developed for detecting the SARS-CoV-2 virus. $^{23-26}$ The fluorophores released by the Cas-proteins could be excited, and the fluorescent emission was used for quantifying the amount of viral sequences. ${ }^{27}$ The NA detection, which incorporates this amplification process to enhance the detection signal, has grown into a mainstream pathogen detection approach with both superior sensitivity and specificity. However, limited by the fluorescence readout method, CRISPR-based detection generally requires the preamplification of target NA or combining multiple crRNAs targeting different viral RNA regions. ${ }^{26,27}$ Biosensors that have higher sensitivity than the direct fluorescence or colorimetric readout scheme could provide fast-response results and capability for POC detection. ${ }^{28}$ Among them, plasmonics, including the localized surface plasmon resonance (LSPR) and surface plasmon polariton (SPP), have become indispensable tools for label-free detection of molecules and quantification of the binding kinetics. ${ }^{29-32}$ Therefore, combining the amplification process and plasmonic detection could further increase the overall performance of biosensing. Meanwhile, the energy losses of plasmonics and the associated heat generation at the nanoscale, known as the plasmonic photothermal (PPT) effect or thermoplasmonics, could benefit a broad range of research and innovation topics, including photothermal-assisted plasmonic sensing (PTAPS). ${ }^{33-35}$ Our recent study proved that the plasmonic biosensor utilizing the dual functions of photonstimulated electromagnetic field and the dissipated local photothermal heat was able to facilitate the specific NA hybridization and achieve a sensitive direct detection of the nonamplified NA sequences of SARS-CoV-2. ${ }^{35}$ Compared with traditional macroscopic heating approaches, the localized thermoplasmonic heating can significantly enhance the reactivity of biomolecules in the vicinity of the LSPR sensing surface by establishing a two-dimensional nanoscale heating zone, while the bulk solution is kept at a lower temperature (room temperature) to suppress the reactivity of biological components. ${ }^{36}$ These findings suggested that the thermoplasmonic and near-field heating also have potential to regulate the biocatalytic behaviors of site-specific nucleases, thereby achieving in situ amplification-based detection on a nanophotonic chip and further improving the sensitivity of the plasmonic biosensor. $^{35}$

In this work, we further extend the utility of the PTAPS biosensing system based on our recently published work ${ }^{35}$ and introduce a thermoplasmonic-assisted dual-mode transducing (TP-DMT) concept, which is able to provide self-validating biosensing readouts for quantifying the viral NA sequence (Figure 1). The gold nanoisland (AuNI) on the sensor chips was employed as a multifunctional medium with the roles of nanoabsorber, nanoheater, and nanotransducer. The thermoplasmonic heating is able to regulate the local biological reactions including the NA sequence hybridization, the endonuclease cleavage, and the cyclic binding-cleavagedissociation (BCD) reaction, while the direct LSPR sensing with the AuNI nanotransducer is able to monitor the nanoscale reactions and provide dual sensitive responses for SARS-CoV-2 quantification. The first amplification-free biosensing readout is

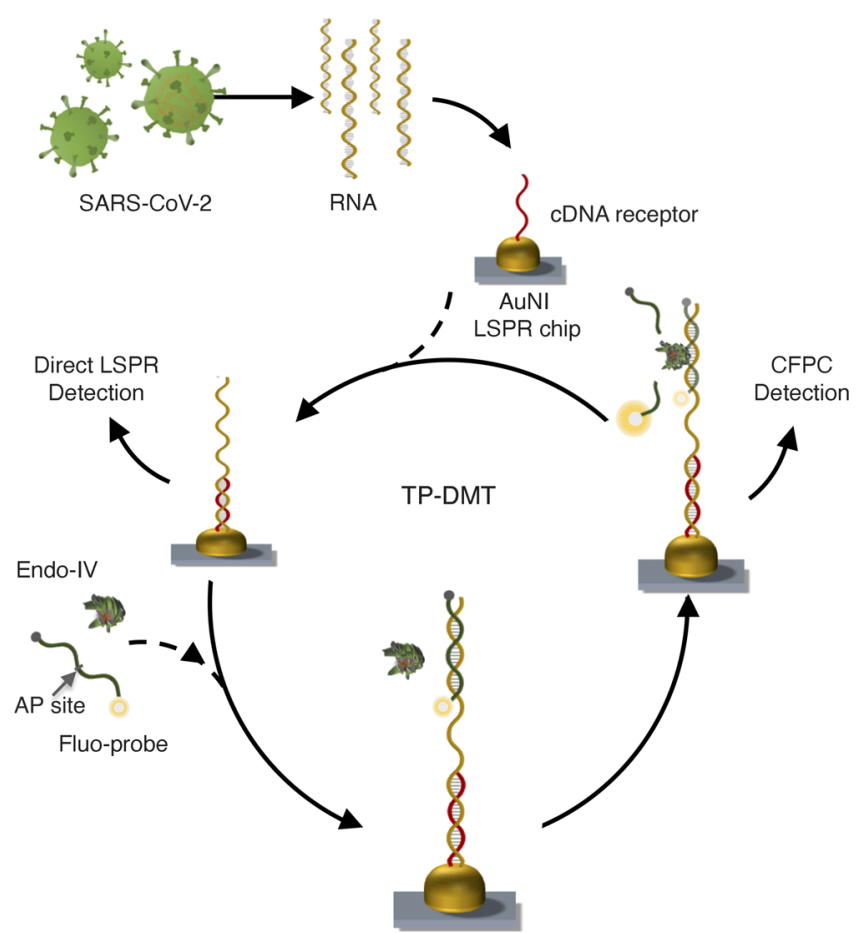

Figure 1. Schematic of TP-DMT viral sensing workflow. The AuNI plasmonic chip functionalized with the complementary DNA receptor can specifically hybridize with the target viral sequence and provide the direct phase detection signal for the first quantification analysis. Subsequently, the AP-site-modified fluorescent probe and Endo-IV are introduced. The Endo-IV is capable of cleaving the AP site after the fluorescent probe hybridizes with the viral sequence. With the assistance of the local PPT heating, the shortened fluorescent probe thermally dehybridizes from the target viral sequence and stimulates the LSPR response as a nonquenched gain medium, thereby providing a highly sensitive secondary CFPC detection signal.

based on the hybridization between the viral target sequences and the functionalized thiol-DNA receptors. With the aid of thermoplasmonics, the two-dimensional AuNI array can provide a suitable local heating condition for the specific hybridization and monitor the real-time molecular binding within the LSPR near-field. The LSPR transducing signal is correlated with the captured NA sequence amount and can be used to evaluate the virus concentration. The limit of detection (LOD) of the direct detection can reach $0.1 \pm 0.04 \mathrm{pM}$. The second LSPR biosensing readout is derived from the cyclic fluorescence probe cleavage (CFPC). Endonuclease IV, a sitespecific nuclease, is utilized to cleave the synthetic apurinic/ apyrimidinic (AP) site when the fluorescent DNA probes hybridize with the target sequences, splitting the long DNA probes into two short strands. Within the local thermoplasmonic field, the two short strands which contain $5^{\prime}$ fluorophore and $3^{\prime}$-quencher termini can dissociate from the target sequence and emit the "switch-on" fluorescent signal. These cyclically released fluorescent probes can stimulate transient and cumulative LSPR responses, thereby providing highly sensitive quantification results with an improved LOD of $0.275 \pm 0.051 \mathrm{fM}$.

\section{RESULTS AND DISCUSSION}

Near-Field Photothermal Effect for Plasmonics Biosensing. The thermoplasmonic effects, which provided the 
A

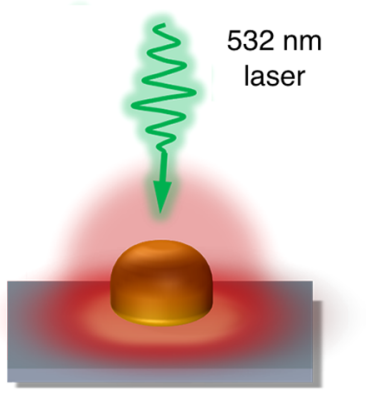

B

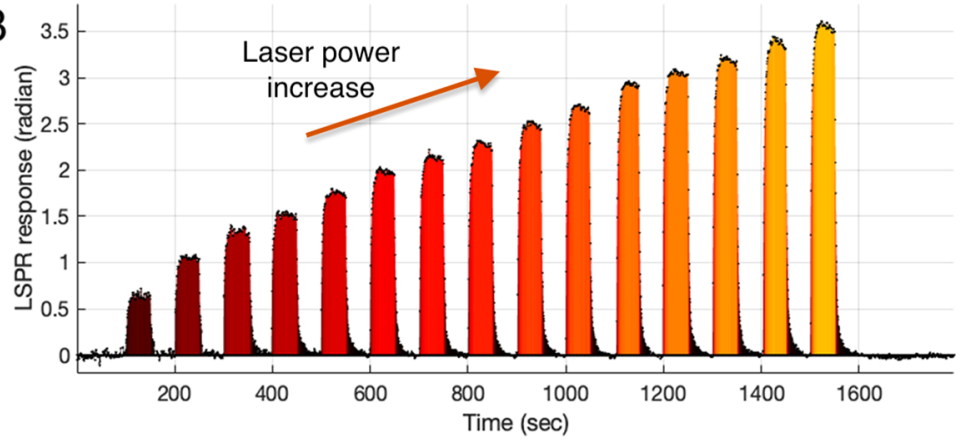

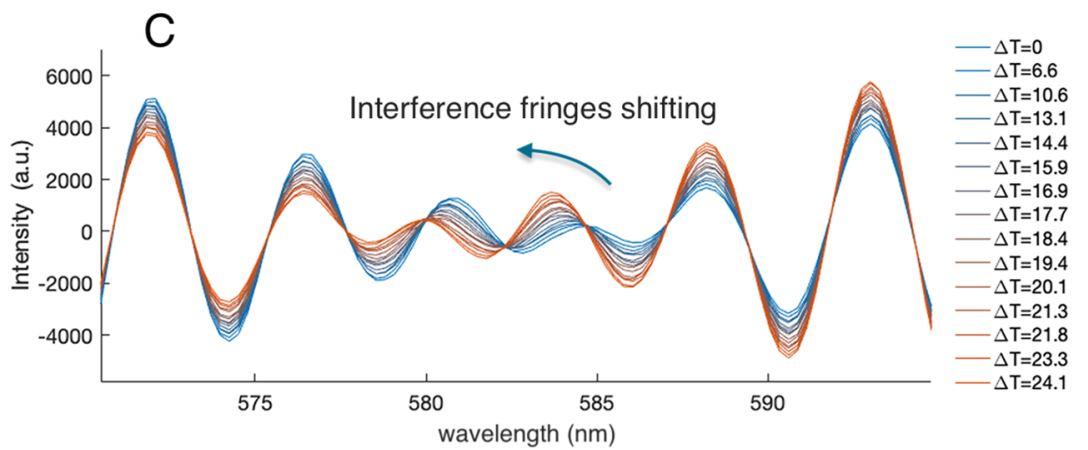

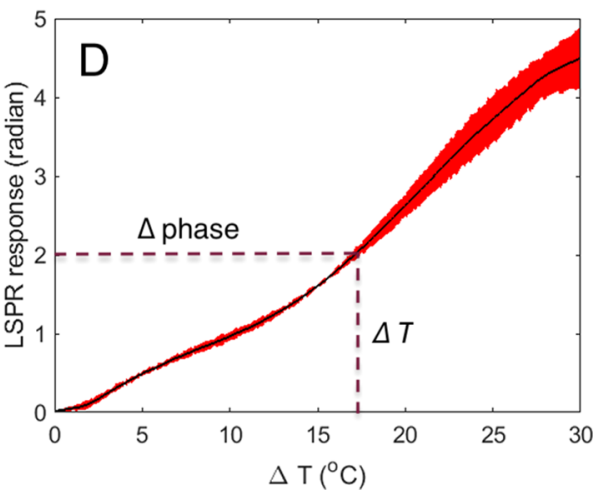

Figure 2. Thermoplasmonic characterization and local temperature calibration. (A) Schematic illustration of the local thermoplasmonics of AuNIs. The $532 \mathrm{~nm}$ laser was matched with the peak absorption of the optimized AuNI chip, which led to a high photothermal efficiency. (B) Real-time differential phase responses stimulated by increased irradiation power. The laser power increased step-by-step from $13.44 \mathrm{~W} /$ $\mathrm{cm}^{2}$ to $94.56 \mathrm{~W} / \mathrm{cm}^{2}$ with 15 complete laser on/off irradiation cycles. (C) Thermoplasmonics of AuNIs led to the interferometric spectra shift, and each fringe with a different phase condition corresponded to a specific local temperature elevation. (D) Correlation curve of photothermal heating and phase change obtained through three repeated temperature calibrations. The solid black curve represents the $\Delta$ phase $-\Delta T$ correlation of mean values, and the red-shaded area represents the standard deviation $( \pm \delta)$ of the corresponding temperature or phase response.
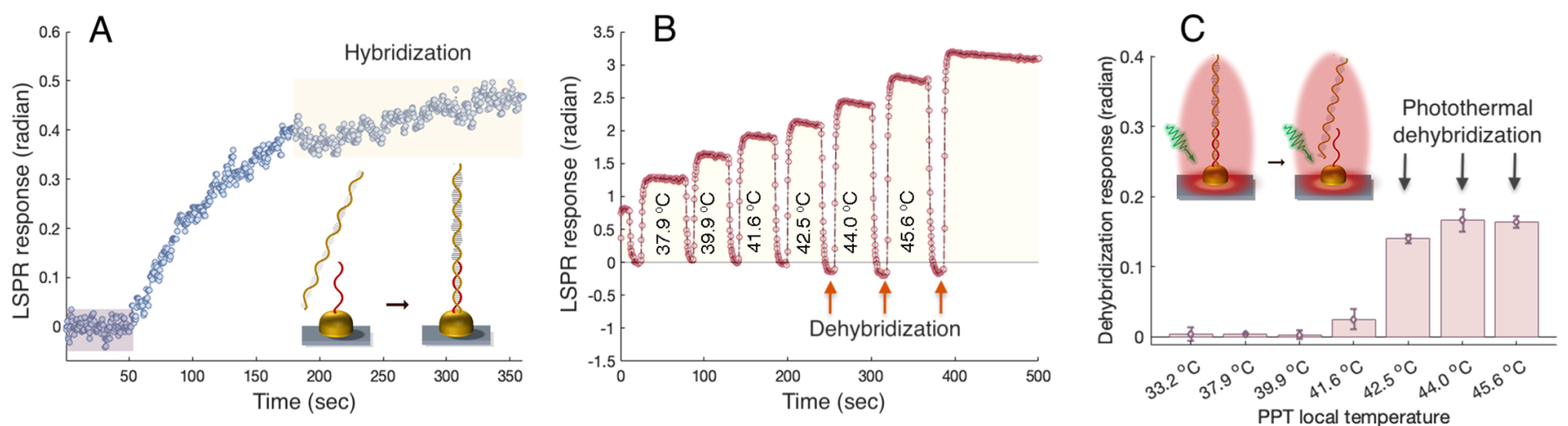

Figure 3. Photothermal hybridization and dehybridization with in situ LSPR phase monitoring. (A) The complementary probe hybridized to a specific position of the NA target (inset), thereby triggering a real-time LSPR phase change. (B) The plasmonic sensor chip loaded with the hybridized NA sequence was treated by a series of photothermal laser on/off cycles with gradually increased power, and the dehybridization (NA dissociation) at different temperatures was investigated. (C) Absolute phase responses caused by NA dehybridization at different temperatures. When the AuNI thermoplasmonic field generated local temperatures higher than $42.5{ }^{\circ} \mathrm{C}$, part of the hybridized complementary strands underwent thermal dissociation (inset).

desired temperature for NA hybridization, Endo-IV cleavage, and cyclic fluorescent probe release, were experimentally characterized by measuring the thermo-induced variation of the refractive index. ${ }^{37}$ The two-dimensional densely distributed nanoabsorbers (AuNI) were initially optimized to have the peak absorption at $532 \mathrm{~nm}$ with a normal incident angle. ${ }^{35,38}$ When irradiated by the homogenized $532 \mathrm{~nm}$ laser (Supplementary Figure S1a), the hot electron-hole pairs were photoexcited within the AuNI nanoabsorbers. Within a time period on the order of nanoseconds, the photoexcited plasmon quickly transferred to the metallic lattice and eventually released the thermal energy to the surrounding water medium through electron-phonon collisions (Figure 2A). ${ }^{39}$ This thermal dissipation process was responsible for a local temperature elevation and a thermally induced variation of the refractive index in the vicinity of the AuNIs. ${ }^{40}$ In the thermoplasmonic and biosensing tests, the homogenized square laser spot fully covered the center region of the 
A
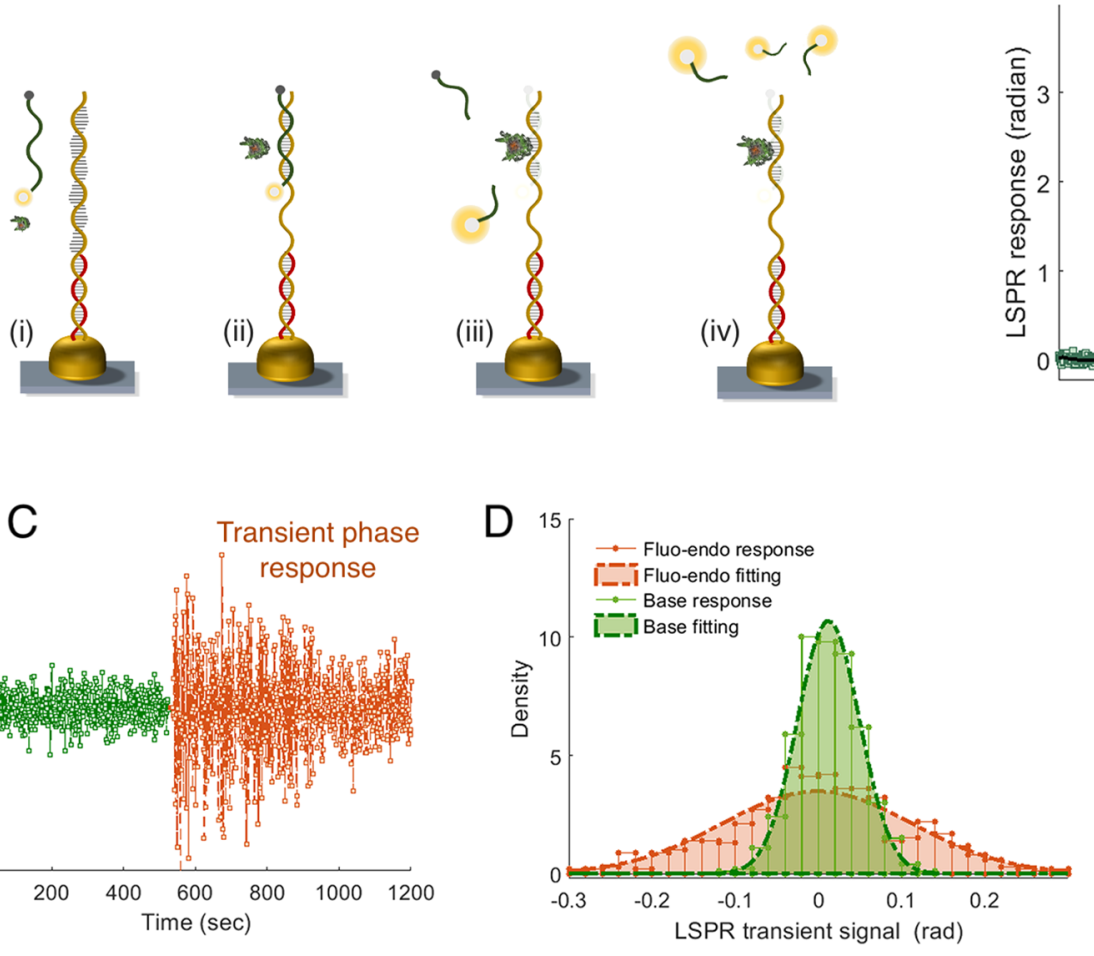

B

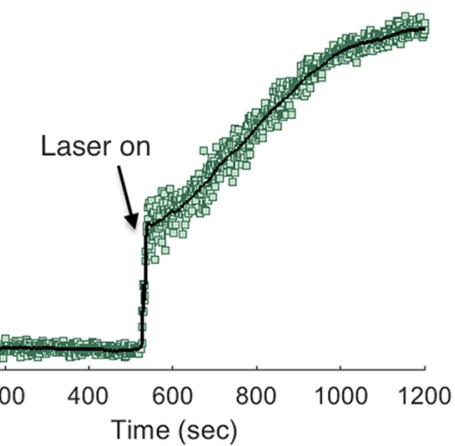

E

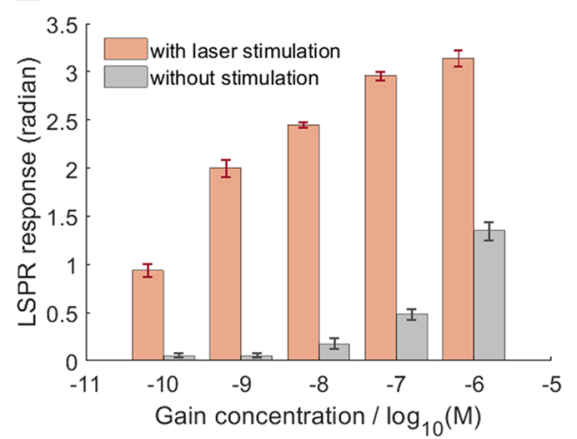

Figure 4. In situ transducing amplification with cyclic Endo-IV reaction. (A) Schematic illustration of the signal amplification cycle with (i) fluorescent probe binding, (ii) AP site cleavage by Endo-IV, (iii) photothermal release of the cleaved short oligos, and (iv) fluorophore accumulation with cyclic cleavage and thermal release reaction. (B) Real-time sensorgram of the cyclic amplification, with a typical laser-off background and an amplified phase response under PTAPS. (C) Transient phase response signal obtained by subtracting the accumulated change. The green scattered dots (1-500 s) represent the background signal without laser irradiation, and the red scattered dots (500-1200 s) represent the transient phase responses stimulated by the near-field cyclic released fluorophores. (D) Phase response distribution of the background and transient signals. (E) Direct detection of gain molecules (rhodamine 6G) to investigate the amplification efficiency of gainassisted plasmonic detection.

microfluidic channel and the transducing region as shown in Supplementary Figure S1b. We believed that the square laser beam $\left(4 \mathrm{~mm}^{2}\right)$ with an even power distribution can provide a uniform thermoplasmonic irradiation in the center sensing region $\left(0.2 \mathrm{~mm}^{2}\right)$. By gradually increasing the irradiation power, a fast and stable in situ temperature adjustment could be achieved (Figure 2B). Simultaneously, we exploited the total internal reflection (TIR) light and LSPR to transduce the spectral phase shift and quantify the variation of the refractive index and local temperature. ${ }^{32}$ According to the interferometric spectra in Figure $2 \mathrm{C}$, the $\mathrm{PPT}$ heating thermally induced a decrease of the local refractive index of the ambient solution and therefore transformed the resonance wavelength shift to a shorter wavelength region. The interference fringe with the smallest amplitude shifted from approximately 585 $\mathrm{nm}$ at room temperature (blue spectrum) to $580 \mathrm{~nm}$ (red spectrum) after thermoplasmonic heating. By using the windowed Fourier transform (WFT) method as described in Supplementary Figure S1c-e and our previous works, the differential phase response was extracted and used for a quantitative real-time retrieval of the local temperature. ${ }^{41,42}$ The detailed temperature characterization and plasmonic heating calibration can be found in Supplementary Figure S2 and our recent work on the PTAPS. ${ }^{35}$ The retrieved relationship between the LSPR phase response ( $\Delta$ phase) and elevated temperature $(\Delta T)$ (Figure $2 \mathrm{D}$ ) was used to estimate the nanoscale temperature around the AuNIs, which regulated the local reactions including the sequence hybridization, endonuclease cleavage, and cyclic BCD reaction.
At a temperature below the melting threshold $\left(T_{\mathrm{m}}\right)$, two complementary NA strands can form a thermodynamically favored double-helix (duplex) structure by establishing the noncovalent and sequence-specific base-pair interaction. ${ }^{43}$ This perfect bonding, known as the NA hybridization, is the molecular basis for many biological processes and NA analytical biotechnologies. ${ }^{44}$ In our first-step hybridization test as shown in Figure 3A, the LSPR differential interferometric phase sensing system probed the on-chip hybridization of two complementary sequences (i.e., the synthetic target viral sequence VTS-nsp13 and its complementary viral receptor specified in Supplementary Table S1). Specifically, the thiolate DNA receptors were covalently immobilized onto the AuNI photonic chips through forming $\mathrm{Au}-\mathrm{S}$ bonds. When the fully matched sequences reached the functionalized biosensing surface, the DNA receptors were able to capture and hybridize with these target sequences as shown in the inset of Figure 3A. This binding reaction changed the local refractive index surrounding the AuNIs and was directly sensed by the LSPR near-field, thereby producing a fast and real-time phase response. ${ }^{42}$ In order to further understand the thermodynamics of the immobilized nucleic acids, we used the PTAPS platform to study the hybridization and dehybridization behaviors. The far-field laser excitation was able to control the near-field thermoplasmonic heating on the AuNI sensor surface to reach the desired temperature, therefore optimizing the hybridization condition for a specific sequence. ${ }^{45}$ Generally, higher temperature can increase the free diffusion of single-stranded NA sequences and make the hybridization 
thermodynamically favorable, thereby improving hybridization kinetics. ${ }^{46}$ However, temperatures approaching the melting threshold cause the dehybridization of double-stranded NA sequences. As shown in Figure 3B, we performed a series of in situ dehybridization tests on the DNA duplexes by gradually increased PPT heating. The thermoplasmonic-induced local temperature gradually increased from $37.9^{\circ} \mathrm{C}$ to $45.6{ }^{\circ} \mathrm{C}$. After each heating cycle, the irradiation laser was switched off, and the local temperature quickly reduced to a typical room temperature at $25^{\circ} \mathrm{C}$. Under this laser-off condition, the realtime phase response with a negative value was recorded and used to determine whether the PPT-induced dehybridization occurred. As shown in Figure 3C, when the thermoplasmonic temperature was below $41.6{ }^{\circ} \mathrm{C}$, the absolute values of the dehybridization responses were weak, which indicated that the NA duplexes maintained the double-stranded state. However, in a PPT near-field with the temperature higher than $42.5^{\circ} \mathrm{C}$, a negative LSPR phase signal appeared (Figure 3B), which indicated that part of the NA duplexes had dehybridized by the AuNI thermoplasmonic field. This dehybridization process was substantial at temperatures higher than $42.5{ }^{\circ} \mathrm{C}$ (Figure 3C) for this target viral sequence, showing that higher temperatures were not favorable for effective in situ detection of the selected viral NA target.

Amplification-Based CFPC Biosensing with Endo-IV. Direct hybridization detection, representing the amplificationfree sensing approach, is able to probe samples quickly with high virus content. ${ }^{7}$ However, when there is only a trace amount of virus in the sample, the detection becomes challenging. Therefore, improving the sensitivity plays a significant role for sensor development. Here, we utilized Endo-IV and the fluorescent probe (22nt) possessing a synthetic AP site for the cyclic gain-assisted amplification and in situ secondary CFPC transducing. Endo-IV plays a crucial role in repairing damaged DNA-containing abasic sites. $^{47}$ It specifically recognizes and cleaves the AP site within a DNA duplex by generating a hydroxyl group at the 3 'terminus. In the CFPC detection, $50 \mu \mathrm{L}$ of freshly prepared reaction solution containing 1 unit of Endo-IV and $1 \mu \mathrm{M}$ fluorescent DNA probes was applied onto the biosensor surface loaded with the captured target sequences. The PPT local heating provided a suitable nanoscale temperature $(T=$ $41.3 \pm 0.2{ }^{\circ} \mathrm{C}$, with $\Delta$ phase $\left.=1.85 \mathrm{rad}\right)$ for both DNA hybridization and enzyme cleavage. On the AuNI surface, the synthesized fluorescent probes first hybridized with the captured sequences in the section that was still single-stranded (Figure 4A, i,ii). The melting temperature $T_{\mathrm{m}}$ of the fluorescent probe was determined to be $54{ }^{\circ} \mathrm{C}$ with nearestneighbor methods (Supplementary Table S1). Then, Endo-IV recognized the AP site in the middle of the probe and cleaved the probe into two short pieces (Figure 4A, ii,iii), which contained an ATTO532 fluorophore and a BHQ1 quencher, respectively. After the cleavage, the two short oligonucleotides possessed much lower melting temperatures at 34 and $30^{\circ} \mathrm{C}$, respectively (Supplementary Table S1). Since the PPTinduced local temperature was evidently higher than their $T_{\mathrm{m}}$ thresholds, the two short oligonucleotides dissociated from the target sequence and departed from the single-stranded viral sequence, leaving it available for the next cyclic $\mathrm{BCD}$ reaction (Figure 4A, iii). The cleavage performance of the Endo-IV on single- and double-stranded sequences with AP sites was further verified with real-time PCR characterization (Supplementary Figure S3). It was found that the Endo-IV was specifically reactive on the double-stranded sequences possessing the synthetic AP sites, while exhibiting an extremely low cleavage rate on the single-stranded probes (control in Supplementary Figure S3). It is worth noting that the thermoplasmonic field by the two-dimensional distributed AuNI array has a small penetration depth into the dielectric media. Therefore, the temperature of the bulk solution is not impacted by the localized heating, and the restriction endonuclease away from the surface has limited reactivity, thereby reducing nonspecific enzymatic reactions. In the CFPC test, the released fluorophores were simultaneously excited by the $532 \mathrm{~nm}$ laser and emitted photons of a low energy (Supplementary Figure S4). Before diffusing into the bulk solution, the released fluorophores were in the vicinity of the AuNI biosensing surface (about $14.9 \mathrm{~nm}$ based on the BDNA structural form). Since the randomly distributed AuNI nanophotonic chip has a broad absorbance superimposing over both the excitation and emission peak of the ATTO532 fluorophore, this distance $(<15 \mathrm{~nm})$ could facilitate the direct energy transfer between the fluorophore and AuNIs, therefore stimulating a transient LSPR phase response through the nearfield interaction as shown in the real-time sensorgram (Figure $4 \mathrm{~B}$ and $\mathrm{C}) .^{48}$ In comparison with the background phase response (without laser excitation) in the first $500 \mathrm{~s}$ of the reaction, the near-field interaction (500 to $1200 \mathrm{~s}$ ) between the released fluorophores (gain molecules) and LSPR AuNIs produced transient phase jumps with high amplitudes. Different from the molecular binding, the instantaneous phase responses caused by this energy transfer and gain medium stimulation demonstrated a wider phase distribution (Figure 4D). As a signal distinct from the background LSPR phase response, this transient phase jump provided qualitative proof to verify the existence of the target sequence.

This cyclic BCD process proceeded continuously on the AuNI biosensor surface under the cooperation of Endo-IV and local thermoplasmonic heating. Subsequently, the fluorescent DNA probes diffused into the bulk solution, and the overall fluorescence intensity gradually increased over time (Figure 4B). The accumulated fluorescent irradiation was able to continuously stimulate the LSPR response as an optically active gain medium. According to the literature, the gainassisted amplification, which is known as the surface plasmon amplification biosensor (SPAB), could amplify the LSPR phase shifting in the TIR light and be used for highly sensitive quantification (Figure 4B). ${ }^{49}$ As a control experiment, we further verified the gain-enhanced detection by using laserassisted detection of rhodamine 6G (R6G) fluorophores in the liquid. As shown in Figure 4E, laser excitation of the photoactive medium (i.e., R6G) significantly amplified the LSPR phase detection response compared to that without laser irradiation. Since the ATTO532 molecules have similar emission properties to R6G (Supplementary Figure S4), this set of control experiments proved that the cumulated fluorescent probes in the liquid effectively provided sensitive and reliable gain-assisted SPAB biosensing results. The CFPC reaction and gain-assisted amplification could only happen when the fluorescent probe, Endo-IV, and photothermal field coexisted (Supplementary Figure S5). Moreover, under the same temperature (corresponding to a constant cleavage rate by the Endo-IV) and time duration, the accumulation rate of the released fluorescent DNA probe was proportional to the number of target NA sequences on the AuNI biosensor chip. ${ }^{27}$ Therefore, the differential CFPC phase response can be used 

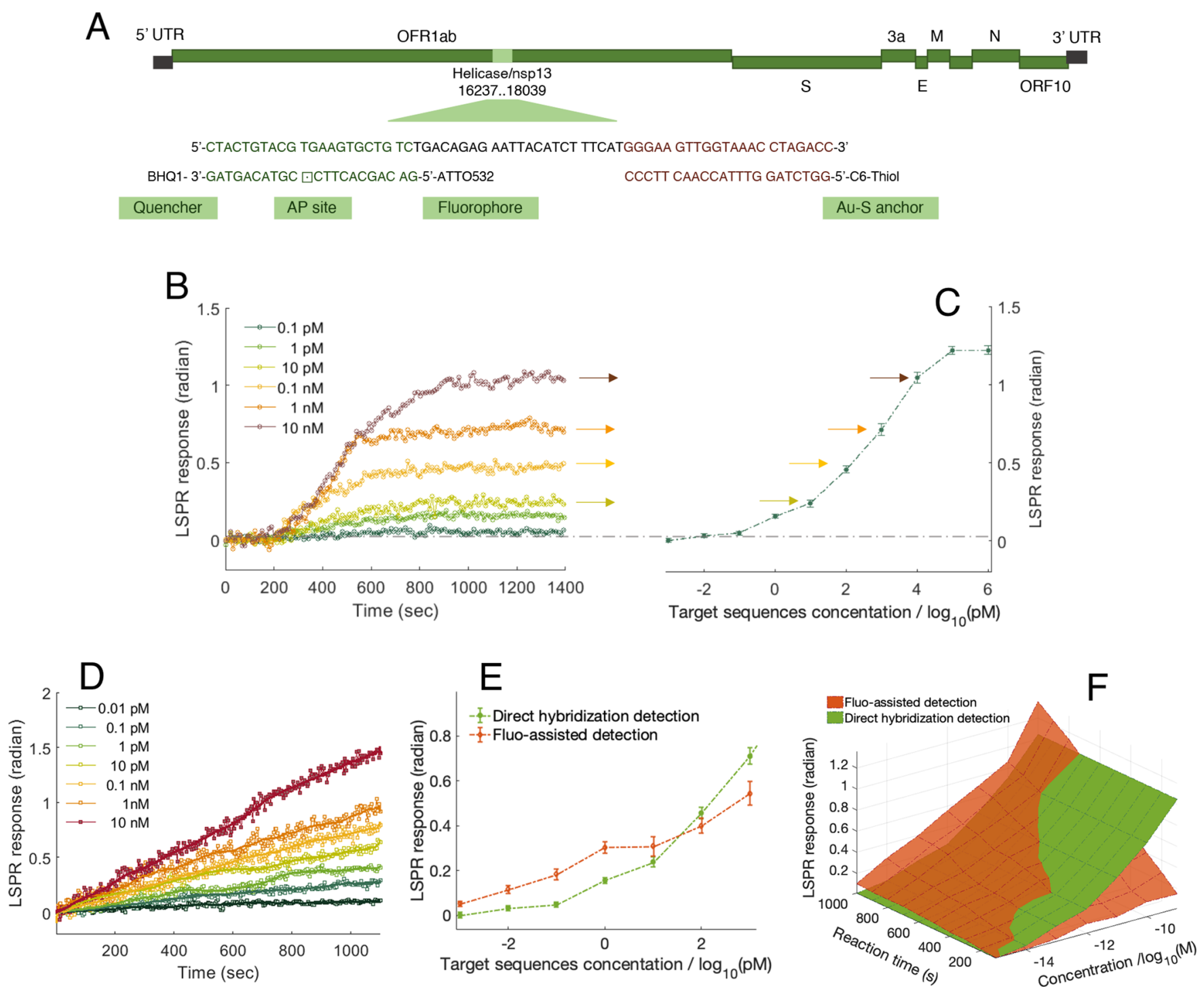

Figure 5. Genomic design and selection of the NA probe/receptor used for TP-DMT detection of SARS-CoV-2, and the analytical sensing performance in the direct and CFPC detections of the viral sequence. (A) Selected NA probe/receptor, viral target sequence, and their relative positions in SARS-CoV-2 (NC_045512.2, GenbBank). (B) Real-time sensorgrams of the target sequence direct detection, with a series of concentrations from $0.1 \mathrm{pM}$ to $10 \mathrm{nM}$. (C) Calibration curve of the direct detection responses versus the target sequence concentrations using the PTAPS. (D) Real-time sensorgrams of the CFPC detection by using the Endo-IV and fluorescent probe, with a series of concentrations from $0.01 \mathrm{pM}$ to $10 \mathrm{nM}$. (E) Comparison of the first-step direct detection (with $800 \mathrm{~s}$ reaction time) and secondary CFPC detection (with $500 \mathrm{~s}$ reaction time). (F) Mapping the detection responses of the CFPC detection as a function of the reaction time and VTS-nsp13 concentration. The green reference surface is the direct detection responses of different NA concentrations with a constant 800 s reaction time.

for the quantitative measurement of the viral NA concentration.

Quantitative Analysis with the Dual-Mode Biosensors. The TP-DMT sensing concept, which combined the direct hybridization and CFPC detections, was first validated with the synthetic viral sequence of SARS-CoV-2. According to the reference SARS-CoV-2 full genomic sequence (NC_045512.2) in GenBank, we selected the viral target sequence in the helicase/nonstructural protein 13 (nsp13) gene region as shown in Figure 5A. This viral target sequence (VTS-nsp13) with a length of 65nt has good representativeness and specificity compared with the corresponding location of six other human coronaviruses (Supplementary Table S2). Based on this target VTS-nsp13 sequence, we further designed the complementary DNA receptor for direct detection of the virus sequence, as well as the AP-site-modified fluorescent probe containing a 5'-ATTO532 head and a $3^{\prime}$-BHQ1 quencher terminus (Figure $5 \mathrm{~A}$ ) for $\mathrm{CFPC}$ reaction. We utilized conventional PCR to detect both the standard SARS-CoV-2 sequences and the selected 65nt VTS-nsp13 sequences, so as to further verify the quality and performance of these synthetic probes and receptors (Supplementary Figures S6 and S7). With the thiol modification, the AuNI biosensing chips were directly functionalized with the viral receptor through $\mathrm{Au}-\mathrm{S}$ covalent bonding, and the detailed optimization of surface functionalization was discussed in our recent PTAPS work. ${ }^{35}$ The functionalized microfluidic AuNI biosensing chip was initially used for the direct viral target sequence quantification. The real-time direct detections of VTS-nsp 13 with a concentration range from $0.1 \mathrm{pM}$ to $10 \mathrm{nM}$ 

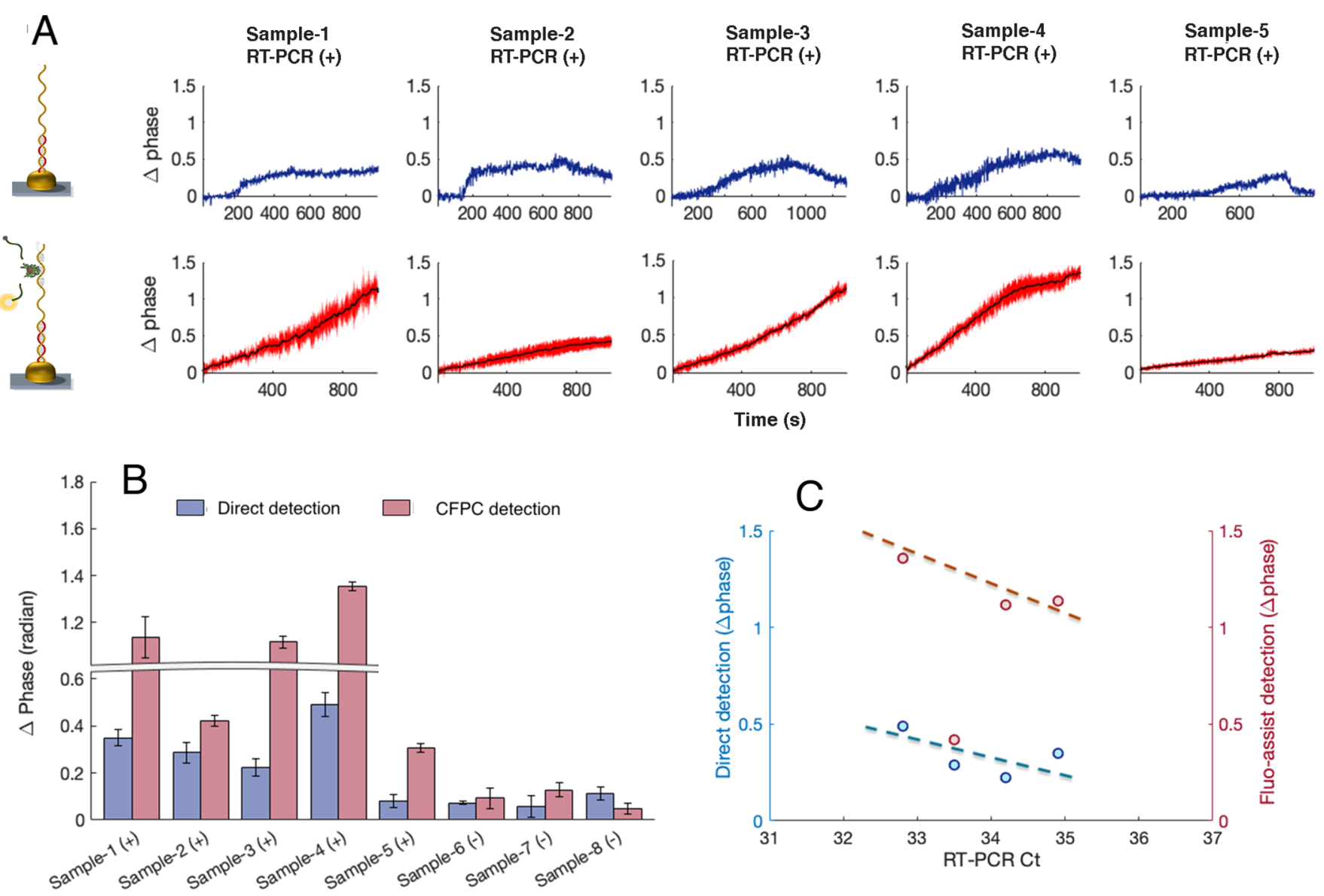

Figure 6. TP-DMT biosensing validation with COVID-19 patient samples. Swab samples from eight patient volunteers were tested using both the direct detection and CFPC detection approaches. (A) Real-time detection of five clinical samples with the direct detection method (the blue curves) and CFPC detection method (the red curves). All patient samples were diluted 50 times with nuclease-free water. (B) Comparison of the LSPR phase responses of the two proposed detection methods. (C) Relationship between the RT-PCR cycle threshold and the responses of the TP-DMT sensor.

are shown in Figure 5B. Prior to the detection, a baseline was established. In each detection, the standard VTS-nsp13 sample was injected into the microfluidic PTAPS detection chamber. The thermoplasmonic assistant unit constructed a uniform two-dimensional photothermal field on the surface of the AuNI sensor, thereby promoting the rapid hybridization between the VTS-nsp13 and the complementary DNA receptor. After the direct hybridization stage, we continued to flush the microfluidic AuNI sensor with nuclease-free water to remove nonspecific binding substances on the surface and acquired the final differential phase responses. The quantification regression curve was obtained by calibrating the final phase responses of the varying VTS-nsp13 sequence concentrations as shown in Figure 5C. The direct sensing system attained the saturation and maximum response condition when the concentration of the VTS-nsp13 sequence reached $100 \mathrm{nM}$. Defining the LOD as the sum of blank measurement responses (i.e., $0.0029 \mathrm{rad}$ ) and triple the standard deviation of the background noises (i.e., $3 \times 0.0031 \mathrm{rad}$ ), we calculated that the LOD of the direct viral sequence detection was about $0.1 \pm$ 0.04 pM (Supplementary Figure S8).

After the direct viral sequence detection, we next used the mixture of Endo-IV and fluorescent probes to perform the secondary CFPC detection. The fluorescent probes initially hybridized to the captured VTS-nsp13 sequences by forming a DNA duplex (Figure 4A, i,ii). Then the Endo-IV recognized the AP site in the DNA probe and cleaved the fluorescent probes into two short strands. Simultaneously, the local thermal field constructed by the homogenized laser beam dissociated the two short strands and "switched-on" the fluorescent gain medium to amplify the plasmonic response, as demonstrated in Figure 4A. As this BCD process can be circularly performed under the thermoplasmonic effect, we observed the LSPR phase response continuously increased (Figure 5D). Different from the hybridization-based direct detection, this gain-assisted CFPC detection signal continued to grow in a linear manner over a long period of time. In the concentration range from $0.01 \mathrm{pM}$ to $10 \mathrm{nM}$, the LSPR phase increment was positively correlated with the concentration of the VTS-nsp13 sequence. We initially set the CFPC reaction time to $500 \mathrm{~s}$, and the regression fitting of the TP-DMT detection is given in Figure 5E. It was found that the CFPC approach reported enhanced phase responses when detecting the viral sequence with concentrations lower than $30 \mathrm{pM}$. Using the same LOD calculation method as described above, we estimate the LOD of CFPC detection as $0.275 \pm 0.051 \mathrm{fM}$ (Supplementary Figure S9), which was more than 2 orders of magnitude lower than that of the direct detection approach.

We subsequently analyzed the CFPC detection responses at different reaction times and further compared them with the direct LSPR hybridization detection at different concentration levels (Figure 5F). Thanks to the cyclic BCD reaction and the 
cumulated gain stimulation, the CFPC-based LSPR detection demonstrated higher sensitivity than the direct detection approach. Especially when detecting the low-concentration samples $(<0.1 \mathrm{pM})$, the CFPC-based detection was capable of providing reliable biosensing results, while the direct detection could hardly report an accurate phase response for viral quantification (Figure 5F). The amplification-based CFPC detection approach outperformed the direct hybridization (amplification-free) detection in the low concentration range, which made the CFPC approach more sensitive. The improved sensitivity of the CFPC detection was at the expense of extended reaction time and extra reagents. Nevertheless, compared with other SARS-CoV-2 biosensors (Supplementary Table S3), the overall biosensing performance of the CFPC detection is still very competitive for fast and sensitive SARSCoV-2 detection. In contrast, the direct detection method could give quantitative test results when analyzing higher concentration samples. In practical applications, when the direct LSPR detection and the CFPC detection both report positive (or negative) results, self-verification of these dualmode readouts is achieved, which delivers a more reliable viral biosensing result (Supplementary Table S4). If the final readout of the direct viral hybridization detection provides negative results or weak positive responses (possibly caused by spurious hybridization or nonspecific bonding), the secondary CFPC could further verify whether the sample contains a trace amount of the viruses. Compared with the conventional PCR approach, the TP-DMT biosensing system can be faster, providing two sensitive readouts within $30 \mathrm{~min}$, and the reverse transcription process can be omitted. Compared with other electrochemical or optical SARS-CoV-2 biosensors, the TPMDT system can implement two interdependent yet different self-validating tests on the single AuNI chip, thereby achieving higher accuracy and reliability.

Clinical-Patient-Sample Test with the TP-DMT Biosensor. To further validate our TP-DMT concept, we next tested clinical swab samples from volunteer patients, including the COVID-19 positive and negative samples determined by the standard reverse transcript RT-PCR (Supplementary Table S5). For each patient sample, we performed the TP-DMT analysis of direct hybridization detection and CFPC amplified detection (Figure 6A and Supplementary Figure S10). In the real-time direct detection of the positive samples (blue curves in Figure 6A), the viral RNA in the 50-fold diluted patient sample in situ hybridized with the viral DNA receptors on the sensor and caused an LSPR phase shift. In the final flushing stage, the phase alteration caused by the bulk refractive index change was further eliminated. The final phase responses were utilized to determine the presence of the SARS-CoV-2 viral RNA in the samples. The decreased phase signal in the flushing step in Figure 6A and Supplementary Figure S10 demonstrated the refractive index changes caused by the testing medium. It is worth noting that flushing with buffer solution when testing clinical or environmental samples was important to eliminate the nonspecific binding events so that the final LSPR detection response was representative of the specific hybridization of the target RNA. In the analysis of the positive samples with a typical viral loading, the direct hybridization detection was capable of providing reliable viral biosensing results. These positive signals were consistent with the RT-PCR detection as given in Table S5. The sample from patient-5 represented a biospecimen with a relatively low viral load. It was confirmed positive for COVID-19 with the highly sensitive Xpert Xpress
SARS-CoV-2 RT-PCR assay running on a GeneXpert System (Cepheid, USA), whereas three other different RT-PCR assays, namely, Cobas SARS-CoV-2 running on a Cobas 6800 system (Roche Diagnostics, Mannheim, Germany), QIAstat-Dx Respiratory 2019-nCoV Panel (Qiagen, Germany), and an in-house-developed RT-PCR method at the Institute of Virology, University of Zurich, all reported negative results. The COVID-19 infection was further verified by testing the serum antibodies, which reported positive results for both IgG and IgA, indicating seroconversion. In the LSPR direct detection of sample-5, only a weak LSPR signal was obtained after the buffer flushing, which was similar to the signal of the negative samples (Supplementary Figure S10). The results demonstrate that the direct detection method was only capable of processing clinical samples with relatively high virus concentrations $\left(>10^{4}\right.$ copies $\left./ \mathrm{mL}\right)$, while low viral load samples need to be further verified by the CFPC biosensing approach.

In the subsequent CFPC test, the SARS-CoV-2 sequences in the positive COVID-19 patient samples initiated the continuous reaction of cleavage and fluorescence enhancement, enabling the LSPR system to report robust phase responses (red curves in Figure 6A). Especially when testing sample-5, a positive CFPC phase response was retrieved, thus confirming the presence of trace virus sequences in the sample. By directly comparing the dual-mode results of TP-DMT (Figure 6B), we found that for both positive and negative samples TP-DMT could provide cross verification and therefore deliver results with high accuracy. For clinical samples with low virus content, e.g., positive patient sample5 , the secondary CFPC detection demonstrated higher sensitivity. By correlating the TP-DMT LSPR sensing results with the RT-PCR threshold cycles (Figure 6C), we further retrieved the quantitative relationship between the dual-mode sensing results and the viral loading. The regressions further proved that direct detection and CFPC detection can effectively perform self-validating dual-mode detection on viral samples. Between them, the direct detection featured better linearity, while the CFPC detection demonstrated better sensitivity.

\section{CONCLUSIONS}

In this study, we present the cleavage-amplification-based plasmonic biosensing approach as an amplification-based detection to identify the presence of the target SARS-CoV-2 virus. As an amplification restrictase, Endo-IV recognized the synthetic AP site and cleaved the fluorescent probes in the hybridized duplex. Within the nanoscale thermoplasmonic field, the shortened probe which contains a fluorophore terminus dissociates from the target viral sequence and emits the "switch-on" fluorescent signal. These cyclically released fluorescent probes stimulated the LSPR response as a photoactive gain medium, thereby providing highly sensitive readouts for quantifying SARS-CoV-2 sequences. Combined with the thermoplasmonics and direct viral sequence detection, the concept of TP-DMT was realized and validated. This TPDMT concept can achieve rapid and reliable viral sequence detection at different concentration levels. Especially for the samples with trace amounts of viruses, this dual-mode detection method can achieve a lower detection limit and higher reliability compared to the direct hybridization approach. Furthermore, we validated our biosensor with clinical samples from COVID-19 patients. This TP-DMT biosensing platform with multifunctional nanotechnologies has 
demonstrated valuable merits for rapid, sensitive, and reliable detection, which has a promising application prospect in realtime virus detection and early warning of SARS-CoV-2. In the context of the COVID-19 pandemic and postpandemic era, this proposed biosensing platform can be a potential tool for fast clinical infection screening and real-time environmental monitoring, therefore contributing to public health interventions and restoring normal production and life.

\section{MATERIALS AND METHODS}

Materials. All chemicals were purchased from commercial suppliers and used without further purification. Nuclease-free water (Sigma-Aldrich) with $50 \mathrm{mM} \mathrm{NaCl}$ was used as the general buffer for dilution of the synthetic viral target sample and patient samples. SARS-CoV-2 target sequences were designed based on the available reference genomes (NC_045512) from GISAID. The selected viral probes and receptors were synthesized and provided by Microsynth (Balgach, Switzerland). Endo-IV (2 U/ $\mu$ L, EN0591, Thermo-Fisher) was used in the CFPC detection to recognize and cleave AP sites of the double-stranded sequences in the reaction buffer (with $50 \mathrm{mM}$ Tris-acetate, $50 \mathrm{mM} \mathrm{NaCl}, 50 \mathrm{mM} \mathrm{KCl}, 1 \mathrm{mM}$ EDTA, $0.05 \mathrm{v} / \mathrm{v} \%$ Triton X-100, $\mathrm{pH} 7.5$ ).

Thermoplasmonic Characterization. The thermoplasmonicinduced local temperature was characterized through measuring the thermo-induced variation of the refractive index. ${ }^{35}$ To calibrate the LSPR phase responses to the alteration of the thermo-induced refractive index, we measured the temperature change and phase response of a water solution in parallel as shown in Supplementary Figure S2. Subsequently, the $532 \mathrm{~nm}$ laser (GML-FN-532 nm-1.5W, CNI, Changchun) with a homogenized beam (Supplementary Figure S1a) was applied on the AuNI sensor chip to construct the local photothermal fields. By adjusting the output power of the laser, the local temperature of the photon-induced heating could be adjusted.

AuNI Biosensor Fabrication and Surface Functionalization. The AuNI sensor chips were synthesized through thermal dewetting of a Au nanofilm (about $5.1 \mathrm{~nm}$ ) at $550{ }^{\circ} \mathrm{C}$ for $3 \mathrm{~h}^{42}$ Subsequently, the PDMS microfluidic channels and AuNI sensor chips were bonded via oxygen plasma. Immobilization of viral thiol-DNA receptors on AuNI sensors followed our previously reported method. ${ }^{35}$ The AuNI sensor surface was functionalized through flowing $100 \mu \mathrm{L}$ of freshly prepared viral sequence receptor $(10 \mu \mathrm{M})$ for 30 min. After flushing with the buffer, the AuNI chips were then rinsed with 6-mercapto-1hexanol $(\mathrm{MCH})$ for $15 \mathrm{~min}$. By thoroughly flushing with the nucleasefree water, the AuNI sensor chips were ready for the TP-DMT detections.

Sequence Hybridization and Dehybridization Tests. In the hybridization tests, $100 \mu \mathrm{L}$ of target sequence samples was injected into the microfluidic AuNI detection chamber; afterward, it was rinsed with a buffer of $50 \mathrm{nM} \mathrm{NaCl}$. The LSPR white-light commonpath interferometric phase detection system transduced the local sequence hybridization in real time. ${ }^{41}$ The AuNI chips containing the hybridized sequence were subsequently used for investigating the in situ PPT-induced dehybridization. In detail, gradually increased laser power was applied onto the AuNI chip. When the local thermoplasmonic temperature was high enough to break the stacking attraction between the complementary bases, we observed the corresponding phase responses in the phase sensorgram.

Detection with the TP-DMT Biosensor. The TP-DMT detection concept included the direct viral sequence detection and the CFPC detection to provide self-validating viral-sensing results. The direct viral sequence detection was based on the hybridization reaction demonstrated above. In addition, the optimized PPT temperature was applied on the AuNI chip to further enhance the hybridization kinetics. In CFPC detection, a $50 \mu \mathrm{L}$ reaction solution containing a unit of Endo-IV and $1 \mu \mathrm{M}$ fluorescent DNA probes was injected into the reaction chamber for the cyclic BCD reaction. The homogenized laser beam provided thermoplasmonic assistance in this reaction process: providing an elevated temperature suitable for EndoIV to cleave the AP sites in the DNA duplex and making the short oligo sequences dissociate from the viral target sequences. The excitation of surface plasmons manifested itself as the phase shift near the resonance wavelength at approximately $584 \mathrm{~nm}$. By employing the windowed Fourier transform calculation, the phase change was extracted from the spectral interferometric patterns and utilized for quantifying the local refractive index change and molecular bindings. ${ }^{41,42}$

Patient Clinical Sample Collection and Preparation. Nasopharyngeal swabs from patients with suspected COVID-19 eluted in universal transport media were collected and analyzed at the University Hospital Zurich. The RNA samples of the patient samples were transported to the ETH laboratory for the TP-DMT studies. Sample RNA of SARS-CoV-2 was analyzed following instructions as described in the FDA-approved protocol with the Xpert Xpress SARSCoV-2 RT-PCR assay running on GeneXpert System (Cepheid, USA). This RT-PCR assay targeted both (N2 and E) nucleic acids. The LOD for this assay was 250 copies $/ \mathrm{mL}$.

Real-Time RT-PCR Assay to Confirm the Viral Fluorescent Probe Performance and Monitoring of Endo-IV Activity. To confirm the viral fluorescent probe performance, a $10 \mu \mathrm{L}$ reaction containing $1 \mu \mathrm{L}$ of SARS-CoV-2 qPCR template standard (HK212669, ORIGENE) or $1 \mu \mathrm{L}$ of VTS-nsp13 sequence, $5 \mu \mathrm{L}$ of $2 \times$ TaqMan master mix was used. qPCR primer mix was used at 5 pmol per reaction and viral fluorescent probe was used at 20 pmol per reaction. Thermal cycling was performed at $95{ }^{\circ} \mathrm{C}$ for $20 \mathrm{~s}$ and then 45 cycles of $95{ }^{\circ} \mathrm{C}$ for $1 \mathrm{~s}$ and $60^{\circ} \mathrm{C}$ for $20 \mathrm{~s}$ by Applied Biosystems ViiA7 instruments (Applied Biosystems, Switzerland). To monitor Endo-IV activity, a $10 \mu \mathrm{L}$ reaction solution that contained $1 \mathrm{U}$ of Endo-IV and 20 pmol of viral fluorescent probes was used to test a series of samples of different concentrations. The fluorescent intensity was monitored at $42{ }^{\circ} \mathrm{C}$ every $20 \mathrm{~s}$ for $600 \mathrm{~s}$ by Applied Biosystems ViiA7 instruments (Applied Biosystems, Switzerland).

Study Approval. No ethical approval for this study was required. Patients gave general consent upon admission to the University Hospital of Zurich allowing reuse of their samples for research purposes.

\section{ASSOCIATED CONTENT}

\section{Supporting Information}

The Supporting Information is available free of charge at https://pubs.acs.org/doi/10.1021/acsnano.1c00957.

Supplementary figures and tables including the laser profile; characterization of local PPT heating; information on designed sequences; comparison of seven human coronaviruses; characterization of Endo-IV cleavage; excitation and emission spectra of fluorescent probes; comparison of the CFPC reactions; standard PCR characterization of viral probe and target sequence; sensor characterizations of the dual-mode detections; approaches for SARS-CoV-2 viral detection; readout analysis; detection of the negative clinical samples; information on clinical patient samples (PDF)

\section{AUTHOR INFORMATION}

\section{Corresponding Authors}

Gerd A. Kullak-Ublick - Department of Clinical Pharmacology and Toxicology, University Hospital Zurich, University of Zürich, Zürich 8091, Switzerland; Mechanistic Safety, CMO \& Patient Safety, Global Drug Development, Novartis Pharma, Basel 4002, Switzerland;

Email: gerd.kullak@usz.ch

Jing Wang - Institute of Environmental Engineering, ETH Zürich, Zürich 8093, Switzerland; Laboratory for Advanced Analytical Technologies, Empa, Swiss Federal Laboratories for Materials Science and Technology, Dübendorf 8600, 
Switzerland; ๑ orcid.org/0000-0003-2078-137X;

Email: jing.wang@ifu.baug.ethz.ch

\section{Authors}

Guangyu Qiu - Institute of Environmental Engineering, ETH Zürich, Zürich 8093, Switzerland; Laboratory for Advanced Analytical Technologies, Empa, Swiss Federal Laboratories for Materials Science and Technology, Dübendorf 8600, Switzerland

Zhibo Gai - Experimental Center, Shandong University of Traditional Chinese Medicine, Jinan 250355, People's Republic of China; Department of Clinical Pharmacology and Toxicology, University Hospital Zurich, University of Zürich, Zürich 8091, Switzerland

Lanja Saleh - Institute of Clinical Chemistry, University Hospital Zurich, University of Zurich, Zurich 8091, Switzerland

Jiukai Tang - Institute of Environmental Engineering, ETH Zürich, Zürich 8093, Switzerland; Laboratory for Advanced Analytical Technologies, Empa, Swiss Federal Laboratories for Materials Science and Technology, Dübendorf 8600, Switzerland

Ting Gui - Experimental Center, Shandong University of Traditional Chinese Medicine, Jinan 250355, People's Republic of China

Complete contact information is available at:

https://pubs.acs.org/10.1021/acsnano.1c00957

\section{Author Contributions}

G.Q., Z.G., G.K.-U., and J.W. conceived the research ideas. G.Q. constructed the PTAPS system in J.W.'s group and conceived the experiments. Z.G. and G.K.-U. contributed to the oligonucleotide design and performed the standard PCR characterization. J.T. contributed to the microfluidic fabrication. L.S. provided and analyzed the clinical human biospecimens. G.Q. conducted the data analysis and wrote the manuscript. J.W. supervised the project. All authors have discussed the results and have given approval to the final version of the manuscripts.

\section{Notes}

The authors declare no competing financial interest.

\section{ACKNOWLEDGMENTS}

This work was supported by the National Research Program (NRP 78 Covid-19, 198258) of the Swiss National Science Foundation (SNSF).

\section{REFERENCES}

(1) Morens, D. M.; Fauci, A. S. Emerging Pandemic Diseases: How We Got to COVID-19. Cell 2020, 182, 1077-1092.

(2) WHO Coronavirus Disease (COVID-19) Dashboard. https:// covid19.who.int/ (accessed Feb 27, 2021).

(3) Aleta, A.; Martin-Corral, D.; Piontti, A. P. Y.; Ajelli, M.; Litvinova, M.; Chinazzi, M.; Dean, N. E.; Halloran, M. E.; Longini, I. M.; Merler, S.; Pentland, A.; Vespignani, A.; Moro, E.; Moreno, Y. Modelling the Impact of Testing, Contact Tracing and Household Quarantine on Second Waves of COVID-19. Nat. Hum. Behav. 2020, 4, 964-971.

(4) Flaxman, S.; Mishra, S.; Gandy, A.; Unwin, H. J. T.; Mellan, T. A.; Coupland, H.; Whittaker, C.; Zhu, H.; Berah, T.; Eaton, J. W.; Monod, M.; Ghani, A. C.; Donnelly, C. A.; Riley, S.; Vollmer, M. A. C.; Ferguson, N. M.; Okell, L. C.; Bhatt, S. Estimating the Effects of Non-Pharmaceutical Interventions on COVID-19 in Europe. Nature 2020, 584, 257-261.
(5) Ong, S. W. X.; Tan, Y. K.; Chia, P. Y.; Lee, T. H.; Ng, O. T.; Wong, M. S. Y.; Marimuthu, K. Air, Surface Environmental, and Personal Protective Equipment Contamination by Severe Acute Respiratory Syndrome Coronavirus 2 (SARS-CoV-2) From a Symptomatic Patient. JAMA 2020, 323, 1610-1612.

(6) Morales-Narvaez, E.; Dincer, C. The Impact of Biosensing in a Pandemic Outbreak: COVID-19. Biosens. Bioelectron. 2020, 163, 112274.

(7) Talebian, S.; Wallace, G. G.; Schroeder, A.; Stellacci, F.; Conde, J. Nanotechnology-Based Disinfectants and Sensors for SARS-CoV-2. Nat. Nanotechnol. 2020, 15, 618-621.

(8) van Doremalen, N.; Bushmaker, T.; Morris, D. H.; Holbrook, M. G.; Gamble, A.; Williamson, B. N.; Tamin, A.; Harcourt, J. L.; Thornburg, N. J.; Gerber, S. I.; Lloyd-Smith, J. O.; de Wit, E.; Munster, V. J. Aerosol and Surface Stability of SARS-CoV-2 as Compared with SARS-CoV-1. N. Engl. J. Med. 2020, 382, 1564-1567.

(9) Morawska, L.; Cao, J. J. Airborne Transmission of SARS-CoV-2: The World Should Face the Reality. Environ. Int. 2020, 139, 105730.

(10) Rahmani, A. R.; Leili, M.; Azarian, G.; Poormohammadi, A. Sampling and Detection of Corona Viruses in Air: A Mini Review. Sci. Total Environ. 2020, 740, 140207.

(11) Corman, V. M.; Landt, O.; Kaiser, M.; Molenkamp, R.; Meijer, A.; Chu, D. K.; Bleicker, T.; Brünink, S.; Schneider, J.; Schmidt, M. L. Detection of 2019 Novel Coronavirus (2019-nCoV) by Real-Time RT-PCR. Eurosurveillance 2020, 25, 2000045.

(12) Wang, W. L.; Xu, Y. L.; Gao, R. Q.; Lu, R. J.; Han, K.; Wu, G. Z.; Tan, W. J. Detection of SARS-CoV-2 in Different Types of Clinical Specimens. J. Am. Med. Assoc. 2020, 323, 1843-1844.

(13) Pan, Y.; Zhang, D.; Yang, P.; Poon, L. L.; Wang, Q. Viral Load of SARS-CoV-2 in Clinical Samples. Lancet Infect. Dis. 2020, 20, 411412 .

(14) Moitra, P.; Alafeef, M.; Dighe, K.; Frieman, M.; Pan, D. Selective Naked-Eye Detection of SARS-CoV-2 Mediated by N Gene Targeted Antisense Oligonucleotide Capped Plasmonic Nanoparticles. ACS Nano 2020, 14, 7617-7627.

(15) Chen, Z.; Zhang, Z.; Zhai, X.; Li, Y.; Lin, L.; Zhao, H.; Bian, L.; Li, P.; Yu, L.; Wu, Y. Rapid and Sensitive Detection of Anti-SARSCoV-2 IgG Using Lanthanide-Doped Nanoparticles-Based Lateral Flow Immunoassay. Anal. Chem. 2020, 92, 7226-7231.

(16) Nachtigall, F. M.; Pereira, A.; Trofymchuk, O. S.; Santos, L. S. Detection of SARS-CoV-2 in Nasal Swabs Using MALDI-MS. Nat. Biotechnol. 2020, 38, 1168-1173.

(17) Seo, G.; Lee, G.; Kim, M. J.; Baek, S.-H.; Choi, M.; Ku, K. B.; Lee, C.-S.; Jun, S.; Park, D.; Kim, H. G. Rapid Detection of COVID19 Causative Virus (SARS-CoV-2) in Human Nasopharyngeal Swab Specimens Using Field-Effect Transistor-Based Biosensor. ACS Nano 2020, 14, 5135-5142.

(18) Funari, R.; Chu, K.-Y.; Shen, A. Q. Detection of Antibodies Against SARS-CoV-2 Spike Protein by Gold Nanospikes in an OptoMicrofluidic Chip. Biosens. Bioelectron. 2020, 169, 112578.

(19) Della Ventura, B.; Cennamo, M.; Minopoli, A.; Campanile, R.; Bolletti Censi, S.; Terracciano, D.; Portella, G.; Velotta, R. Colorimetric Test for Fast Detection of SARS-CoV-2 in Nasal and Throat Swabs. ACS Sens 2020, 5, 3043-3048.

(20) Baker, A. N.; Richards, S.-J.; Guy, C. S.; Congdon, T. R.; Hasan, M.; Zwetsloot, A. J.; Gallo, A.; Lewandowski, J. z. R.; Stansfeld, P. J.; Straube, A. The SARS-COV-2 Spike Protein Binds Sialic Acids and Enables Rapid Detection in a Lateral Flow Point of Care Diagnostic Device. ACS Cent. Sci. 2020, 6, 2046-2052.

(21) Grant, B. D.; Anderson, C. E.; Williford, J. R.; Alonzo, L. F.; Glukhova, V. A.; Boyle, D. S.; Weigl, B. H.; Nichols, K. P. SARS-CoV2 Coronavirus Nucleocapsid Antigen-Detecting Half-Strip Lateral Flow Assay toward the Development of Point of Care Tests Using Commercially Available Reagents. Anal. Chem. 2020, 92, 1130511309.

(22) Ravi, N.; Cortade, D. L.; Ng, E.; Wang, S. X. Diagnostics for SARS-CoV-2 Detection: A Comprehensive Review of the FDA-EUA COVID-19 Testing Landscape. Biosens. Bioelectron. 2020, 165, 112454. 
(23) Joung, J.; Ladha, A.; Saito, M.; Kim, N.-G.; Woolley, A. E.; Segel, M.; Barretto, R. P.; Ranu, A.; Macrae, R. K.; Faure, G. Detection of SARS-CoV-2 with SHERLOCK One-Pot Testing. N. Engl. J. Med. 2020, 383, 1492-1494.

(24) Patchsung, M.; Jantarug, K.; Pattama, A.; Aphicho, K.; Suraritdechachai, S.; Meesawat, P.; Sappakhaw, K.; Leelahakorn, N.; Ruenkam, T.; Wongsatit, T. Clinical Validation of a Cas13-Based Assay for the Detection of SARS-CoV-2 RNA. Nat. Biomed. Eng. 2020, 4, 1140-1149.

(25) Ma, P. X.; Meng, Q. Z.; Sun, B. Q.; Zhao, B.; Dang, L.; Zhong, M. T.; Liu, S. Y.; Xu, H. T.; Mei, H.; Liu, J.; Chi, T.; Yang, G.; Liu, M.; Huang, X. X.; Wang, X. J. MeCas12a, a Highly Sensitive and Specific System for COVID-19 Detection. Adv. Sci. 2020, 7, 2001300.

(26) Broughton, J. P.; Deng, X. D.; Yu, G. X.; Fasching, C. L.; Servellita, V.; Singh, J.; Miao, X.; Streithorst, J. A.; Granados, A.; Sotomayor-Gonzalez, A.; Zorn, K.; Gopez, A.; Hsu, E.; Gu, W.; Miller, S.; Pan, C. Y.; Guevara, H.; Wadford, D. A.; Chen, J. S.; Chiu, C. Y. CRISPR-Cas12-Based Detection of SARS-CoV-2. Nat. Biotechnol. 2020, 38, 870-874.

(27) Fozouni, P.; Son, S.; de León Derby, M. D.; Knott, G. J.; Gray, C. N.; D’Ambrosio, M. V.; Zhao, C.; Switz, N. A.; Kumar, G. R.; Stephens, S. I. Amplification-Free Detection of SARS-CoV-2 with CRISPR-Cas13a and Mobile Phone Microscopy. Cell 2021, 184, 323-333.

(28) Udugama, B.; Kadhiresan, P.; Kozlowski, H. N.; Malekjahani, A.; Osborne, M.; Li, V. Y. C.; Chen, H. M.; Mubareka, S.; Gubbay, J. B.; Chan, W. C. W. Diagnosing COVID-19: The Disease and Tools for Detection. ACS Nano 2020, 14, 3822-3835.

(29) Zhang, P.; Ma, G.; Dong, W.; Wan, Z.; Wang, S.; Tao, N. Plasmonic Scattering Imaging of Single Proteins and Binding Kinetics. Nat. Methods 2020, 17, 1010-1017.

(30) Brolo, A. G. Plasmonics for Future Biosensors. Nat. Photonics 2012, 6, 709-713.

(31) Wojtowicz, W. M.; Vielmetter, J.; Fernandes, R. A.; Siepe, D. H.; Eastman, C. L.; Chisholm, G. B.; Cox, S.; Klock, H.; Anderson, P. W.; Rue, S. M.; Miller, J. J.; Glaser, S. M.; Bragstad, M. L.; Vance, J.; Lam, A. W.; Lesley, S. A.; Zinn, K.; Garcia, K. C. A Human IgSF CellSurface Interactome Reveals a Complex Network of Protein-Protein Interactions. Cell 2020, 182, 1027-1043.

(32) Qiu, G.; Thakur, A.; Xu, C.; Ng, S. P.; Lee, Y.; Wu, C. M. L. Detection of Glioma-Derived Exosomes with the Biotinylated Antibody-Functionalized Titanium Nitride Plasmonic Biosensor. Adv. Funct. Mater. 2019, 29, 1806761.

(33) Baffou, G.; Cichos, F.; Quidant, R. Applications and Challenges of Thermoplasmonics. Nat. Mater. 2020, 19, 946-958.

(34) You, M.; Li, Z.; Feng, S.; Gao, B.; Yao, C.; Hu, J.; Xu, F. Ultrafast Photonic PCR Based on Photothermal Nanomaterials. Trends Biotechnol. 2020, 38, 637-649.

(35) Qiu, G.; Gai, Z.; Tao, Y.; Schmitt, J.; Kullak-Ublick, G. A.; Wang, J. Dual-Functional Plasmonic Photothermal Biosensors for Highly Accurate Severe Acute Respiratory Syndrome Coronavirus 2 Detection. ACS Nano 2020, 14, 5268-5277.

(36) Baffou, G.; Quidant, R. Thermo-Plasmonics: Using Metallic Nanostructures as Nano-Sources of Heat. Laser Photonics Rev. 2013 $7,171-187$

(37) Zhang, D. L.; Lan, L.; Bai, Y. R.; Majeed, H.; Kandel, M. E.; Popescu, G.; Cheng, J. X. Bond-Selective Transient Phase Imaging via Sensing of the Infrared Photothermal Effect. Light: Sci. Appl. 2019, 8, $1-12$.

(38) Qiu, G. Y.; Ng, S. P.; Wu, C. M. L. Bimetallic Au-Ag Alloy Nanoislands for Highly Sensitive Localized Surface Plasmon Resonance Biosensing. Sens. Actuators, B 2018, 265, 459-467.

(39) Brongersma, M. L.; Halas, N. J.; Nordlander, P. PlasmonInduced Hot Carrier Science and Technology. Nat. Nanotechnol. 2015, 10, 25-34.

(40) Jauffred, L.; Samadi, A.; Klingberg, H.; Bendix, P. M.; Oddershede, L. B. Plasmonic Heating of Nanostructures. Chem. Rev. 2019, 119, 8087-8130.
(41) Ng, S. P.; Loo, F. C.; Wu, S. Y.; Kong, S. K.; Wu, C. M. L.; Ho, H. P. Common-Path Spectral Interferometry with Temporal Carrier for Highly Sensitive Surface Plasmon Resonance Sensing. Opt. Express 2013, 21, 20268-20273.

(42) Qiu, G. Y.; Ng, S. P.; Wu, C. M. L. Differential Phase-Detecting Localized Surface Plasmon Resonance Sensor with Self-Assembly Gold Nano-Islands. Opt. Lett. 2015, 40, 1924-1927.

(43) Kyogoku, Y.; Lord, R. C.; Rich, A. Hydrogen Bonding Specificity of Nucleic Acid Purines and Pyrimidines in Solution. Science 1966, 154, 518-520.

(44) Wang, J. From DNA Biosensors to Gene Chips. Nucleic Acids Res. 2000, 28, 3011-3016.

(45) Reismann, M.; Bretschneider, J. C.; von Plessen, G.; Simon, U. Reversible Photothermal Melting of DNA in DNA-Gold-Nanoparticle Networks. Small 2008, 4, 607-610.

(46) Zhang, J. X.; Fang, J. Z.; Duan, W.; Wu, L. R.; Zhang, A. W.; Dalchau, N.; Yordanov, B.; Petersen, R.; Phillips, A.; Zhang, D. Y. Predicting DNA Hybridization Kinetics from Sequence. Nat. Chem. 2018, 10, 91-98.

(47) Hosfield, D. J.; Guan, Y.; Haas, B. J.; Cunningham, R. P.; Tainer, J. A. Structure of the DNA Repair Enzyme Endonuclease IV and Its DNA Complex: Double-Nucleotide Flipping at Abasic Sites and Three-Metal-Ion Catalysis. Cell 1999, 98, 397-408.

(48) Moskowitz, J.; Geddes, C. D. The Inverse Relationship between Metal-Enhanced Fluorescence and Fluorophore-Induced Plasmonic Current. J. Phys. Chem. Lett. 2020, 11, 8145-8151.

(49) Ng, S. P.; Yip, Y. Y.; Wu, C.-M. L. Biosensing with GainAssisted Surface Plasmon-Polariton Amplifier: A Computational Investigation. Sens. Actuators, B 2015, 210, 36-45. 